\title{
On the uniqueness of uniform norms and $C^{*}$-norms
}

\author{
by \\ P. A. DabHi and H. V. Dedania (Vallabh Vidyanagar)
}

\begin{abstract}
We prove that a semisimple, commutative Banach algebra has either exactly one uniform norm or infinitely many uniform norms; this answers a question asked by S. J. Bhatt and H. V. Dedania [Studia Math. 160 (2004)]. A similar result is proved for $C^{*}$-norms on $*$-semisimple, commutative Banach $*$-algebras. These properties are preserved if the identity is adjoined. We also show that a commutative Beurling *-algebra $L^{1}(G, \omega)$ has exactly one uniform norm if and only if it has exactly one $C^{*}$-norm; this is not true in arbitrary $*$-semisimple, commutative Banach $*$-algebras.
\end{abstract}

1. Introduction. A uniform norm on an algebra $\mathcal{A}$ is a (not necessarily complete) algebra norm $|\cdot|$ on $\mathcal{A}$ satisfying the square property

$$
\left|x^{2}\right|=|x|^{2} \quad(x \in \mathcal{A}) \text {. }
$$

Note that any two equivalent uniform norms on $\mathcal{A}$ are identical. A Banach algebra $\mathcal{A}$ has the unique uniform norm property (UUNP) if it admits exactly one uniform norm; in this case the spectral radius $r(\cdot)$ on $\mathcal{A}$ is the only uniform norm. This property was introduced and extensively studied by S. J. Bhatt and H. V. Dedania (see $[2-6]$ ). It was proved in $[2$, Theorem 2] that a commutative Beurling algebra $L^{1}(G, \omega)$ has either exactly one uniform norm or infinitely many uniform norms. We extend this result to all semisimple, commutative Banach algebras (see Theorem 2.2(i) below). This result simplifies the proof of [6, Theorem 3.1] which states that a non-unital, semisimple, commutative Banach algebra $\mathcal{A}$ has UUNP if and only if its unitization $\mathcal{A}_{e}$ has UUNP. Using these results we also give a correct proof of [4, Theorem 6.1] which states that if the multiplier algebra $M(\mathcal{A})$ has UUNP, then $\mathcal{A}$ has UUNP.

A $C^{*}$-norm on a $*$-algebra $\mathcal{B}$ is a (not necessarily complete) algebra norm $|\cdot|$ on $\mathcal{B}$ satisfying the $C^{*}$-property

$$
\left|x^{*} x\right|=|x|^{2} \quad(x \in \mathcal{B}) .
$$

2000 Mathematics Subject Classification: 46J05, 46K05, 22D15.

Key words and phrases: commutative Banach algebra, commutative Banach $*$-algebra, uniform norm, $C^{*}$-norm, multiplier algebra, commutative Beurling algebra. 
Like uniform norms, any two equivalent $C^{*}$-norms on $\mathcal{B}$ are identical. A Banach *-algebra $\mathcal{B}$ has the unique $C^{*}$-norm property $\left(U C^{*} N P\right)$ if it admits exactly one $C^{*}$-norm. This property was first studied by B. A. Barnes in [1]. Here we shall prove that a $*$-semisimple, commutative Banach $*$-algebra has either exactly one $C^{*}$-norm or infinitely many $C^{*}$-norms. B. A. Barnes exhibited in [1, Example 4.4] a non-unital, *-semisimple, commutative Banach *-algebra $\mathcal{B}$ such that $\mathcal{B}$ has $\mathrm{UC}^{*} \mathrm{NP}$ but its unitization $\mathcal{B}_{e}$ does not have $\mathrm{UC}^{*} \mathrm{NP}$. However, this is false because of our Corollary 2.3 below which states that $\mathcal{B}$ has $\mathrm{UC}^{*} \mathrm{NP}$ if and only if $\mathcal{B}_{e}$ has $\mathrm{UC}^{*} \mathrm{NP}$ at least for the commutative case. We also exhibit a gap in the arguments of [1, Example 4.4].

In an arbitrary $*$-semisimple, commutative Banach $*$-algebra, the UUNP is stronger than the $\mathrm{UC}^{*} \mathrm{NP}$. However, we prove that they are equivalent in a commutative Beurling *-algebra $L^{1}(G, \omega)$.

2. Main results. Throughout, $\mathcal{A}$ is a semisimple, commutative Banach algebra with the complete norm $\|\cdot\|, r_{\mathcal{A}}(\cdot)(=r(\cdot))$ is the spectral radius on $\mathcal{A}, \triangle(\mathcal{A})$ is the Gel'fand space of $\mathcal{A}$, and $\widehat{x}: \triangle(\mathcal{A}) \rightarrow \mathbb{C}, \varphi \mapsto \varphi(x)$, is the Gel'fand transform of an element $x$ in $\mathcal{A}$. The identity element of $\mathcal{A}$ (if it exists) is denoted by 1 . If $\mathcal{A}$ is non-unital, we take $\mathcal{A}_{e}:=\mathcal{A} \times \mathbb{C}$ with co-ordinatewise linear operations and the ring multiplication being $(x, \alpha)(y, \beta)=(x y+\alpha y+\beta x, \alpha \beta)$. The complete norm $\|\cdot\|$ is extended to $\mathcal{A}_{e}$ by $\|(x, \alpha)\|=\|x\|+|\alpha|$. For a closed subset $F$ of $\triangle(\mathcal{A})$, define

$$
|x|_{F}=|\widehat{x}|_{F}:=\sup \{|\widehat{x}(\varphi)|: \varphi \in F\} \quad(x \in \mathcal{A}) .
$$

Then $|\cdot|_{F}$ is a uniform seminorm on $\mathcal{A}$ and it is dominated by the spectral radius $r(\cdot)$; the seminorm $|\cdot|_{F}$ is a uniform norm on $\mathcal{A}$ if and only if $F$ is a set of uniqueness for $\mathcal{A}$, that is, for any $x \in \mathcal{A}, \varphi(x)=0(\varphi \in F)$ implies $x=0$. On the other hand, let $|\cdot|$ be a uniform norm on $\mathcal{A}$. Set $F:=\{\varphi \in \triangle(\mathcal{A}): \varphi$ is $|\cdot|$-continuous $\}$. Then $F$ is the largest closed subset of $\triangle(\mathcal{A})$ such that $|x|=|x|_{F}(x \in \mathcal{A})$.

Throughout, $\mathcal{B}$ stands for a $*$-semisimple, commutative Banach $*$-algebra. A complex homomorphism $\varphi \in \triangle(\mathcal{B})$ is self-adjoint if $\varphi\left(x^{*}\right)=\overline{\varphi(x)}(x \in \mathcal{B})$. Let $\widetilde{\triangle}(\mathcal{B}):=\{\varphi \in \triangle(\mathcal{B}): \varphi$ is self-adjoint $\}$. Then it is easy to see that $\widetilde{\triangle}(\mathcal{B})$ is closed in $\triangle(\mathcal{B})$. If $F$ is a closed subset of $\widetilde{\triangle}(\mathcal{B})$ and if $F$ is a set of uniqueness for $\mathcal{B}$, then $|\cdot|_{F}$ is a $C^{*}$-norm on $\mathcal{B}$. Conversely, if $|\cdot|$ is a $C^{*}$-norm on $\mathcal{B}$, then there exists a largest closed subset $F$ of $\widetilde{\triangle}(\mathcal{B})$ such that $|x|=|x|_{F}(x \in \mathcal{B})$. Now it is clear that every $C^{*}$-norm is a uniform norm.

THEOREM 2.1 .

(i) Let $\mathcal{A}$ be non-unital. Then $\mathcal{A}$ has infinitely many uniform norms if and only if $\mathcal{A}_{e}$ has infinitely many uniform norms. 
(ii) Let $\mathcal{B}$ be non-unital. Then $\mathcal{B}$ has infinitely many $C^{*}$-norms if and only if $\mathcal{B}_{e}$ has infinitely many $C^{*}$-norms.

Proof. (i) Let $|\cdot|_{1}$ and $|\cdot|_{2}$ be two distinct uniform norms on $\mathcal{A}$. For $i=1,2$, define

$$
|(x, \alpha)|_{i, \text { op }}:=\sup \left\{|x y+\alpha y|_{i}: y \in \mathcal{A},|y|_{i} \leq 1\right\} \quad\left((x, \alpha) \in \mathcal{A}_{e}\right) .
$$

Then both $|\cdot|_{1 \text {,op }}$ and $|\cdot|_{2 \text {,op }}$ are uniform norms on $\mathcal{A}_{e}$, and they are distinct as they are distinct on $\mathcal{A}$ [6, Proposition 2.2]. Thus if $\mathcal{A}$ has infinitely many uniform norms, then so does $\mathcal{A}_{e}$. Now suppose that $|\cdot|_{1}$ and $|\cdot|_{2}$ are two distinct uniform norms on $\mathcal{A}_{e}$. For $i=1,2$, define

$$
F_{i}=\left\{\varphi \in \triangle\left(\mathcal{A}_{e}\right): \varphi \text { is }|\cdot|_{i} \text {-continuous }\right\} .
$$

Then $F_{1} \neq F_{2}$. Without loss of generality assume that $F_{2} \backslash F_{1} \neq \emptyset$. Choose $\varphi \in F_{2} \backslash F_{1}$. We may assume that $\varphi \neq \varphi_{\infty}$. Then $\varphi$ is not $|\cdot|_{1}$-continuous. So there exists a sequence $\left\{\left(x_{k}, \alpha_{k}\right)\right\}$ in $\mathcal{A}_{e}$ such that $\left|\left(x_{k}, \alpha_{k}\right)\right|_{1} \leq 1$ and $\left|\varphi\left(\left(x_{k}, \alpha_{k}\right)\right)\right| \rightarrow \infty$ as $k \rightarrow \infty$. Since $\varphi \neq \varphi_{\infty}$, there exists $b \in \mathcal{A}$ such that $\varphi(b) \neq 0$. Then $\left|x_{k} b+\alpha_{k} b\right|_{1} \leq\left|\left(x_{k}, \alpha_{k}\right)\right|_{1}|b|_{1} \leq|b|_{1}$ and $\left|\varphi\left(x_{k} b+\alpha_{k} b\right)\right|$ $=\left|\varphi\left(\left(x_{k}, \alpha_{k}\right)\right)\right||\varphi(b)| \rightarrow \infty$ as $k \rightarrow \infty$. Therefore $\left|x_{k} b+\alpha_{k} b\right|_{2} \geq$ $\left|\varphi\left(x_{k} b+\alpha_{k} b\right)\right| \rightarrow \infty$ as $k \rightarrow \infty$. So $|\cdot|_{1} \neq|\cdot|_{2}$ on $\mathcal{A}$.

Now suppose that $\mathcal{A}_{e}$ has infinitely many distinct uniform norms. Let $\left\{|\cdot|_{i}: i \in \mathbb{N}\right\}$ be an infinite collection of distinct uniform norms on $\mathcal{A}_{e}$. For $i \in \mathbb{N}$, define

$$
F_{i}:=\left\{\varphi \in \triangle\left(\mathcal{A}_{e}\right): \varphi \text { is }|\cdot|_{i} \text {-continuous }\right\} .
$$

Since $|\cdot|_{i} \neq|\cdot|_{j}(i \neq j)$, we must have $F_{i} \neq F_{j}(i \neq j)$. First, assume that $\varphi_{\infty} \in F_{i}$ for infinitely many $i$ 's. In this case, we may assume that $\varphi_{\infty} \in F_{i}$ for all $i$. Then, by the argument as in the paragraph above, we can show that $|\cdot|_{i} \neq \mid \cdot{ }_{j}$ on $\mathcal{A}$. Thus $\mathcal{A}$ has infinitely many uniform norms. Second, assume that $\varphi_{\infty} \in F_{i}$ for finitely many $i$ 's only. In this case, we may assume that $\varphi_{\infty} \notin F_{i}$ for all $i$. Then, by the same argument as in the paragraph above, one can show that $\mathcal{A}$ has infinitely many uniform norms.

(ii) This follows from (i) and the fact that every $C^{*}$-norm is a uniform norm on a commutative Banach $*$-algebra.

It was asked in $[2$, p. 179] whether there exists $\mathcal{A}$ with finitely many uniform norms. Part (i) of the next result settles this question in the negative.

THEOREM 2.2 .

(i) $\mathcal{A}$ has either exactly one uniform norm or infinitely many uniform norms.

(ii) $\mathcal{B}$ has either exactly one $C^{*}$-norm or infinitely many $C^{*}$-norms.

Proof. (i) First, assume that $\mathcal{A}$ is unital. Let $|\cdot|$ be a uniform norm on $\mathcal{A}$ and suppose $|\cdot| \neq r_{\mathcal{A}}(\cdot)$. Set $F:=\{\varphi \in \triangle(\mathcal{A}): \varphi$ is $|\cdot|$-continuous $\}$. Then $F$ 
is a compact subset of $\triangle(\mathcal{A})$ and $|\cdot|=|\cdot|_{F}$ on $\mathcal{A}$. Certainly, $|\cdot| \leq r_{\mathcal{A}}(\cdot) \leq\|\cdot\|$ on $\mathcal{A}$. Since $|\cdot| \neq r_{\mathcal{A}}(\cdot), F \neq \triangle(\mathcal{A})$. Set $U=\triangle(\mathcal{A}) \backslash F$.

We claim that $U$ is not closed in $\triangle(\mathcal{A})$. Suppose that, if possible, $U$ is closed in $\triangle(\mathcal{A})$. Then, by the Shilov Idempotent Theorem, $\chi_{U} \in \widehat{\mathcal{A}}$, i.e., there exists $x_{0} \in \mathcal{A}$ such that $\widehat{x}_{0}=\chi_{U}$. Then $x_{0} \neq 0$. On the other hand, $\left|x_{0}\right|=\left|\widehat{x}_{0}\right|_{F}=\left|\chi_{U}\right|_{F}=0$. Since $|\cdot|$ is a norm on $\mathcal{A}, x_{0}=0$, which is a contradiction. This proves our claim.

Since $\triangle(\mathcal{A})$ is Hausdorff and since $U$ is not closed, $U$ must be an infinite set. Let $\left\{\varphi_{j}: j \in \mathbb{N}\right\}$ be an infinite subset of $U$. Set $F_{n}:=F \cup\left\{\varphi_{1}, \ldots, \varphi_{n}\right\}$ $(n \in \mathbb{N})$. Then $F_{n}$ is a compact subset of $\triangle(\mathcal{A})$ and $|\cdot|_{F_{n}}$ gives a uniform norm on $\mathcal{A}$ because $|\cdot| \leq|\cdot|_{F_{n}}$. Take any $n \in \mathbb{N}$. Then $\varphi_{i} \neq \varphi_{n}(1 \leq i<n)$. So there exist $a_{1}, \ldots, a_{n-1}$ in $\mathcal{A}$ such that $\varphi_{i}\left(a_{i}\right)=0$ while $\varphi_{n}\left(a_{i}\right) \neq 0$ $(1 \leq i<n)$. Hence $\varphi_{i}\left(a_{1} \ldots a_{n-1}\right)=0$ and $\varphi_{n}\left(a_{1} \ldots a_{n-1}\right) \neq 0$. Therefore, there is $x \in \mathcal{A}$ such that $\varphi_{i}(x)=0(1 \leq i<n)$ and $\varphi_{n}(x)=1$. Since $\varphi_{n}$ is not $|\cdot|_{F}$-continuous, there exists a sequence $\left(x_{j}\right)$ in $\mathcal{A}$ such that $\left|x_{j}\right|_{F} \leq 1$ and $\left|\varphi_{n}\left(x_{j}\right)\right| \rightarrow \infty$ as $j \rightarrow \infty$. Now $\varphi_{i}\left(x x_{j}\right)=0(1 \leq i<n, j \in \mathbb{N})$. This implies that for $1 \leq i<n$ and $j \in \mathbb{N}$,

$$
\left|x x_{j}\right|_{F_{i}}=\sup \left\{\left|\varphi\left(x x_{j}\right)\right|: \varphi \in F_{i}\right\}=\sup \left\{\left|\varphi\left(x x_{j}\right)\right|: \varphi \in F\right\}=\left|x x_{j}\right|_{F}
$$

and $\left|x x_{j}\right|_{F_{n}} \geq\left|\varphi_{n}\left(x x_{j}\right)\right|=\left|\varphi_{n}\left(x_{j}\right)\right| \rightarrow \infty$ as $j \rightarrow \infty$. Hence, $|\cdot|_{F_{n}} \neq|\cdot|_{F_{i}}$ $(1 \leq i<n)$. Since $n \in \mathbb{N}$ was arbitrary, $\mathcal{A}$ has infinitely many distinct uniform norms.

Second, assume that $\mathcal{A}$ is non-unital. Suppose $\mathcal{A}$ does not have exactly one uniform norm. Let $|\cdot|_{1}$ and $|\cdot|_{2}$ be two distinct uniform norms on $\mathcal{A}$. Then, by [6, Proposition 2.2], we get two distinct uniform norms on $\mathcal{A}_{e}$. By Theorem 2.1(i), $\mathcal{A}$ has infinitely many uniform norms. This proves (i).

(ii) First, assume that $\mathcal{B}$ is unital. Let $|\cdot|$ be a $C^{*}$-norm on $\mathcal{B}$ and $|\cdot| \neq m(\cdot)$, where $m(x)=\sup \{|\varphi(x)|: \varphi \in \widetilde{\triangle}(\mathcal{B})\} \quad(x \in \mathcal{B})$, that is, the largest $C^{*}$-norm on $\mathcal{B}$. Set $F:=\{\varphi \in \widetilde{\triangle}(\mathcal{B}): \varphi$ is $|\cdot|$-continuous $\}$. Then $F$ is closed and hence compact in $\widetilde{\triangle}(\mathcal{B})$. Also,

$$
|x|=|x|_{F}=|\widehat{x}|_{F} .
$$

Certainly, $|\cdot| \leq m(\cdot) \leq\|\cdot\|$ on $\mathcal{B}$. Since $|\cdot| \neq m(\cdot), F \neq \widetilde{\triangle}(\mathcal{B})$. Set $U=\widetilde{\triangle}(\mathcal{B}) \backslash F$. Then $U$ is not closed in $\widetilde{\triangle}(\mathcal{B})$. Indeed, if $U$ is closed in $\widetilde{\triangle}(\mathcal{B})$, then $U$ is closed in $\triangle(\mathcal{B})$ because $\widetilde{\triangle}(\mathcal{B})$ is closed in $\triangle(\mathcal{B})$. Then, by the Shilov Idempotent Theorem, $\chi_{U} \in \widehat{\mathcal{B}}$, i.e., there exists $x_{0} \in \mathcal{B}$ such that $\widehat{x}_{0}=\chi_{U}$. Hence $x_{0} \neq 0$ and $\left|x_{0}\right|=\left|\widehat{x}_{0}\right|_{F}=\left|\chi_{U}\right|_{F}=0$. This is a contradiction because $|\cdot|$ is a norm on $\mathcal{B}$.

Now as in (i) above, we can show that $\mathcal{B}$ has either exactly one $C^{*}$-norm or infinitely many $C^{*}$-norms. This remains true in non-unital $\mathcal{B}$ due to Theorem 2.1(ii). Hence (ii) is proved. 
Part (i) of the next corollary was proved in [6, Theorem 3.1]. However, Theorems 2.1(i) and 2.2(i) simplify its proof. Corollary 2.3(ii) exhibits that Example 4.4 given by B. A. Barnes in [1] is false. It was argued there that $C^{*}(A) \cong C^{*}(B)$ because $A$ is $\gamma_{B^{-}}$dense in $B$; but $A$ is, in fact, a proper, $\gamma_{B}$-closed, *-ideal in $B$.

Corollary 2.3.

(i) Let $\mathcal{A}$ be non-unital. Then $\mathcal{A}$ has UUNP if and only if $\mathcal{A}_{e}$ has UUNP.

(ii) Let $\mathcal{B}$ be non-unital. Then $\mathcal{B}$ has $U C^{*} N P$ if and only if $\mathcal{B}_{e}$ has $U C^{*} N P$.

Proof. (i) Assume that $\mathcal{A}$ has UUNP. By Theorems 2.2(i) and 2.1(i), $\mathcal{A}_{e}$ does not have infinitely many uniform norms. So, by Theorem 2.1(i), $\mathcal{A}_{e}$ has UUNP. The converse follows by similar arguments.

(ii) This is in the line of (i).

A multiplier on $\mathcal{A}$ is a linear map $T: \mathcal{A} \rightarrow \mathcal{A}$ satisfying $(T x) y=$ $x(T y)=T(x y)(x, y \in \mathcal{A})$. By [8, Theorem 1.1.1], $T$ is automatically continuous. Let $M(\mathcal{A})$ denote the algebra of all multipliers on $\mathcal{A}$. Then $M(\mathcal{A})$ is a semisimple, commutative Banach algebra with the operator norm $\|T\|=\sup \{\|T x\|: x \in \mathcal{A},\|x\| \leq 1\}$. For $a \in \mathcal{A}$, let $L_{a}: \mathcal{A} \rightarrow \mathcal{A}$ be the multiplication operator $L_{a}(x)=a x(x \in \mathcal{A})$. Then $a \mapsto L_{a}$ is a one-one continuous homomorphism of $\mathcal{A}$ onto the ideal $\left\{L_{a}: a \in \mathcal{A}\right\}$ of $M(\mathcal{A})$. Hence we shall identify $\mathcal{A}$ with that ideal. The Gel'fand space $\triangle(M(\mathcal{A}))$ is a disjoint union of the Gel'fand space $\triangle(\mathcal{A})$ and $h(\mathcal{A})$, the hull of $\mathcal{A}$ in $\triangle(M(\mathcal{A}))$ (see [8, Theorem 1.4.3]).

The next theorem was proved in [4, Theorem 6.1]. However, the proof given there is not correct. In [4, Theorem 6.1], it is not clear why $|\cdot|_{r}$ is a uniform norm on $M(\mathcal{A})$. Here we apply a different technique.

TheOREM 2.4. If $M(\mathcal{A})$ has UUNP, then $\mathcal{A}$ has UUNP.

Proof. It is sufficient to prove that if $\mathcal{A}$ has infinitely many uniform norms, then so does $M(\mathcal{A})$. It is enough to show that any uniform norm on $\mathcal{A}$ can be extended to a uniform norm on $M(\mathcal{A})$.

Let $|\cdot|$ be a uniform norm on $\mathcal{A}$. Then there is a closed subset $F$ of $\triangle(\mathcal{A})$ such that $|\cdot|=|\cdot|_{F}$ on $\mathcal{A}$. Let $\widetilde{F}:=F \cup h(\mathcal{A})$. Define

$$
|T|_{\widetilde{F}}:=\sup \{|\varphi(T)|: \varphi \in \widetilde{F}\} \quad(T \in M(\mathcal{A})) .
$$

It is clear that $|\cdot|_{\widetilde{F}}$ is a uniform seminorm on $M(\mathcal{A})$ and $|\cdot|_{\widetilde{F}}=|\cdot|$ on $\mathcal{A}$. So all we need to prove is that $|\cdot|_{\widetilde{F}}$ is a norm on $M(\mathcal{A})$.

Suppose $|T|_{\widetilde{F}}=0$; then $\left.\widehat{T}\right|_{h(\mathcal{A})}=0$ and $\left.\widehat{T}\right|_{F}=0$. Let $\psi \in \triangle(\mathcal{A}) \backslash F$. Then there is $x \in \mathcal{A}$ such that $\psi(x)=1$. Since $\mathcal{A}$ is an ideal in $M(\mathcal{A}), x T \in \mathcal{A}$. Now $\widehat{(x T)}(\varphi)=\widehat{x}(\varphi) \widehat{T}(\varphi)=0(\varphi \in F)$. Therefore $|x T|=0$. Since $|\cdot|$ is a 
norm on $\mathcal{A}, x T=0$. So $0=\widehat{(x T)}(\psi)=\widehat{x}(\psi) \widehat{T}(\psi)=\widehat{T}(\psi)$. Thus $\left.\widehat{T}\right|_{\triangle(\mathcal{A})}=0$ and $\left.\widehat{T}\right|_{h(\mathcal{A})}=0$, so $\widehat{T}=0$ on $\triangle(M(\mathcal{A}))$. Since $M(\mathcal{A})$ is semisimple, $T=0$. Thus $|\cdot|_{\widetilde{F}}$ is a uniform norm on $M(\mathcal{A})$.

We remark that the converse of Theorem 2.4 is not true; for example, if $G$ is a non-discrete, locally compact, abelian group, then the group algebra $L^{1}(G)$ has UUNP but its multiplier algebra $M\left(L^{1}(G)\right) \cong M(G)$ does not have UUNP (see [4, p. 233]).

Let $G$ be a Hausdorff, locally compact, abelian topological group with the binary operation being addition. The Haar measure on $G$ is denoted by $m$. A weight on $G$ is a measurable function $\omega: G \rightarrow \mathbb{R}$ such that $0<\omega(s+t) \leq$ $\omega(s) \omega(t)(s, t \in G)$. The weight $\omega$ on $G$ is symmetric if $\omega(-s)=\omega(s)(s \in G)$. Define

$$
L^{1}(G, \omega):=\left\{f: G \rightarrow \mathbb{C}: f \text { is measurable and } \int_{G}|f| \omega d m<\infty\right\} .
$$

For $f, g \in L^{1}(G, \omega)$, define the convolution product $f * g$ as follows:

$$
(f * g)(s):=\int_{G} f(s-t) g(t) d m(t) \quad(s \in G) .
$$

Then $L^{1}(G, \omega)$ is a commutative Banach algebra with the convolution product and the norm being $\|f\|_{\omega}:=\int_{G}|f(s)| \omega(s) d m(s)$; it is called a Beurling algebra. By [3, Theorem 1], $L^{1}(G, \omega)$ is semisimple. Note that if $G$ is not abelian, then it is not known whether or not the Beurling algebra $L^{1}(G, \omega)$ is always semisimple (see [7, p. 175]). If the weight $\omega$ is symmetric, then the Beurling algebra $L^{1}(G, \omega)$ is in fact a $*$-semisimple Banach $*$-algebra with respect to the involution $f^{*}(s)=\overline{f(-s)}(s \in G)$.

An $\omega$-bounded generalized character on $G$ is a continuous homomorphism $\alpha:(G,+) \rightarrow(\mathbb{C} \backslash\{0\}, \times)$ such that $|\alpha(s)| \leq \omega(s)(s \in G)$. Let $H(G, \omega)$ be the set of all $\omega$-bounded generalized characters endowed with the compact-open topology. Let $\widehat{G}$ be the dual group of $G$. Then $H(G, \omega) \cong \triangle\left(L^{1}(G, \omega)\right)$ via the mapping $\alpha \mapsto \varphi_{\alpha}$, where

$$
\varphi_{\alpha}(f)=\int_{G} f(s) \alpha(s) d m(s) \quad\left(f \in L^{1}(G, \omega)\right) .
$$

If the weight $\omega$ on $G$ is symmetric, then $\omega(s) \geq 1(s \in G), \widehat{G} \subset H(G, \omega)$ and $\varphi_{\alpha}$ is self-adjoint if and only if $\alpha \in \widehat{G}$. Hence $\widetilde{\triangle}\left(L^{1}(G, \omega)\right) \cong \widehat{G}$ (see [2] and [4]). In an arbitrary $*$-semisimple, commutative Banach $*$-algebra, UUNP implies $\mathrm{UC}^{*} \mathrm{NP}$; but the converse is not true (see [4, Proposition 2.10]). However, here we show that the converse is true in the case of $L^{1}(G, \omega)$. To prove this we shall need the following simple lemma. 
Lemma 2.5. Let $\omega$ be a symmetric weight on $G$ and $H(G, \omega) \neq \widehat{G}$. Then there exists $t \in G$ such that

$$
\sum_{n \in \mathbb{Z}} \frac{\log \omega(n t)}{1+n^{2}}=\infty .
$$

Proof. Since $\omega$ is symmetric, $\omega(s) \geq 1(s \in G)$. Hence $\widehat{G} \subset H(G, \omega)$. Choose $\alpha \in H(G, \omega) \backslash \widehat{G}$. Then there exists $t \in G$ such that $|\alpha(t)| \neq 1$. Since $1=\alpha(0)=\alpha(t) \alpha(-t)$, we may assume that $|\alpha(t)|>1$. Since $\omega(s) \geq 1$ $(s \in G)$, we have

$$
\begin{aligned}
\sum_{n \in \mathbb{Z}} \frac{\log \omega(n t)}{1+n^{2}} & \geq \sum_{n \in \mathbb{N}} \frac{\log \omega(n t)}{1+n^{2}} \geq \sum_{n \in \mathbb{Z}} \frac{\log |\alpha(n t)|}{1+n^{2}} \\
& \geq \sum_{n \in \mathbb{N}} \frac{\log |\alpha(t)|^{n}}{1+n^{2}}=\log |\alpha(t)| \sum_{n \in \mathbb{N}} \frac{n}{1+n^{2}}=\infty
\end{aligned}
$$

THEOREM 2.6. Let $\omega$ be a symmetric weight on $G$. Then $L^{1}(G, \omega)$ has $U U N P$ if and only if it has $U C^{*} N P$.

Proof. Since $\omega$ is symmetric, $\widehat{G} \subset H(G, \omega)$. Assume that $L^{1}(G, \omega)$ has UUNP. Because every $C^{*}$-norm on a commutative Banach *-algebra is a uniform norm it is clear that $L^{1}(G, \omega)$ has $\mathrm{UC}^{*} \mathrm{NP}$.

For the converse, assume that $L^{1}(G, \omega)$ has UC*NP. First we claim that $H(G, \omega)=\widehat{G}$. Suppose, if possible, $H(G, \omega) \neq \widehat{G}$. Then by Lemma 2.5 , there exists $t \in G$ such that

$$
\sum_{n \in \mathbb{Z}} \frac{\log \omega(n t)}{1+n^{2}}=\infty .
$$

Set $U=\{\theta \in \widehat{G}: \operatorname{Re} \theta(t)>0\}=\{\theta \in \widehat{G}:|\theta(t)-1|<\sqrt{2}\}$. Then $1_{G} \in U$ and so $U$ is a non-empty, open subset of $\widehat{G}$. Since $\widehat{G}$ is locally compact, there is a non-empty open subset $V$ of $\widehat{G}$ such that $V \subset \bar{V} \subset U$. Set $F:=\widehat{G} \backslash V$. Then $F$ is closed in $\widehat{G}$ and hence in $H(G, \omega)$. Define $|f|_{F}:=\sup \{|\varphi(f)|: \varphi \in F\}$ $\left(f \in L^{1}(G, \omega)\right)$. It is clear that $|\cdot|_{F}$ is a $C^{*}$-seminorm on $L^{1}(G, \omega)$. We show that it is in fact a $C^{*}$-norm on $L^{1}(G, \omega)$. Suppose that, if possible, there exists $f \in L^{1}(G, \omega)$ such that $f \neq 0$ and $|f|_{F}=0$. Then supp $\widehat{f} \subset \bar{V} \subset U$. So, as in the proof of [9, Theorem A.1.13],

$$
\sum_{n \in \mathbb{Z}} \frac{\log \omega(n t)}{1+n^{2}}<\infty,
$$

which is a contradiction. Hence $|\cdot|_{F}$ is a $C^{*}$-norm on $L^{1}(G, \omega)$. As in the proof of $\left[2\right.$, Theorem 2] we can show that $|\cdot|_{F} \neq|\cdot|_{\widehat{G}}$. This means that $L^{1}(G, \omega)$ does not have $\mathrm{UC}^{*} \mathrm{NP}$, which is again a contradiction. This proves our claim, i.e., $H(G, \omega)=\widehat{G}$. Hence $L^{1}(G, \omega)$ is hermitian. So, by [4, Proposition 2.10(2)(i)], $L^{1}(G, \omega)$ has UUNP. 
Acknowledgements. The authors are grateful to Prof. S. J. Bhatt for reading this manuscript and to the referee for giving fruitful suggestions. They also acknowledge the financial support through UGC-SAP-DRS grant No. F.510/5/DRS/2004 (SAP-I) provided to the Department of Mathematics, Sardar Patel University.

\section{References}

[1] B. A. Barnes, The properties of *-regularity and uniqueness of $C^{*}$-norm in general *-algebras, Trans. Amer. Math. Soc. 279 (1983), 841-859.

[2] S. J. Bhatt and H. V. Dedania, Beurling algebras and uniform norms, Studia Math. 160 (2004), 179-183.

[3] -, -, A Beurling algebra is semisimple: An elementary proof, Bull. Austral. Math. Soc. 66 (2002), 91-93.

[4] -, -, Banach algebras with unique uniform norm II, Studia Math. 147 (2001), 211-235.

[5] -, -, Banach algebras with unique uniform norm, Proc. Amer. Math. Soc. 124 (1996), 579-584.

[6] -, -, Uniqueness of the uniform norm and adjoining identity in Banach algebras, Proc. Indian Acad. Sci. Math. Sci. 105 (1995), 405-409.

[7] H. G. Dales and A. T.-M. Lau, The second duals of Beurling algebras, Mem. Amer. Math. Soc. 177 (2005), no. 836.

[8] R. Larsen, An Introduction to the Theory of Multipliers, Springer, Berlin, 1971.

[9] H. Reiter and J. D. Stegeman, Classical Harmonic Analysis and Locally Compact Groups, London Math. Soc. Monogr. 22, Clarendon Press, Oxford, 2000.

Department of Mathematics

Sardar Patel University

Vallabh Vidyanagar 388120

Gujarat, India

E-mail: lightatinfinite@gmail.com

hvdedania@yahoo.com

Received July 11, 2008

Revised version December 3, 2008 